\title{
Evaluation of National Influenza Sentinel Surveillance System in Nigeria, Jan-Dec 2014
}

\author{
Amaka P. Onyiah*1, ${ }^{\star}$, Muhammad S. Balogun', Adebayo A. Adedeji ${ }^{2}$ and Patrick M. \\ Nguku ${ }^{1}$
}

${ }^{1}$ Nigeria Field Epidemiology and Laboratory Training Program, Abuja, Nigeria; ${ }^{2}$ National Influenza Sentinel Surveillance, Abuja, Nigeria

\section{Objective}

To assess the performance of the surveillance system and identify factors affecting the performance.

\section{Introduction}

National Influenza Sentinel Surveillance (NISS) was established in Nigeria in 2006 to monitor influenza occurrence in humans in Nigeria and provide a foundation for detecting outbreaks of novel strains of influenza. Surveillance for influenza-like illness (ILI) and severe acute respiratory infection (SARI) is carried out in 4 sentinel sites. Specimens and epidemiological data are collected and transported 4 days a week from the sentinel sites to the National Influenza Reference Laboratory. At the laboratory, they are tested for influenza $A$ and $B$ viruses and further subtyped if positive for influenza A virus.

\section{Methods}

Surveillance facilitators from the 4 functional sentinel sites were interviewed via telephone while oral interviews were conducted with laboratory staff. Information on NISS structure and management of data and specimens were collected. NISS protocol was reviewed, and surveillance data from January to December 2014 were analysed using Epi Info software. CDC Updated guidelines for evaluating public health surveillance systems was used to guide the evaluation.

\section{Results}

NISS activities are funded through a cooperative agreement with the US Centres for Disease Control and Prevention (CDC). Of specimens sent from the sentinel sites, $68 \%$ reached the laboratory within 48 hours. Specimen processing in the laboratory took an average of 23 days. Reasons given for the delay include late distribution of supplies from the laboratory to sentinel sites and stock-out of working materials at the laboratory. Surveillance activities were disrupted in the month of July 2014 because of health workers' strike. The predictive value positive was $8.7 \%$. Of submitted case investigation forms, $73.7 \%$ were completely filled. The testing algorithm and NISS protocol have been reviewed (addition of more variables on co-morbidities, age classification of patients seen daily at the sentinel health facilities, and analysis of more influenza subtypes at the laboratory) over the years.

\section{Conclusions}

The NISS is useful in characterizing influenza epidemiology in Nigeria. It is simple, flexible, and acceptable to the stakeholders. However, there was delay in sending of specimens to the laboratory and timely processing of specimens received. The observed delay may undermine its ability to detect early unusual patterns of morbidity and mortality due to influenza or signal the beginning of influenza season. The overdependence of the surveillance activities on donorfunding is a threat to its sustainability. We recommend improvement of logistics management and encouragement of local partnerships for sustainability.

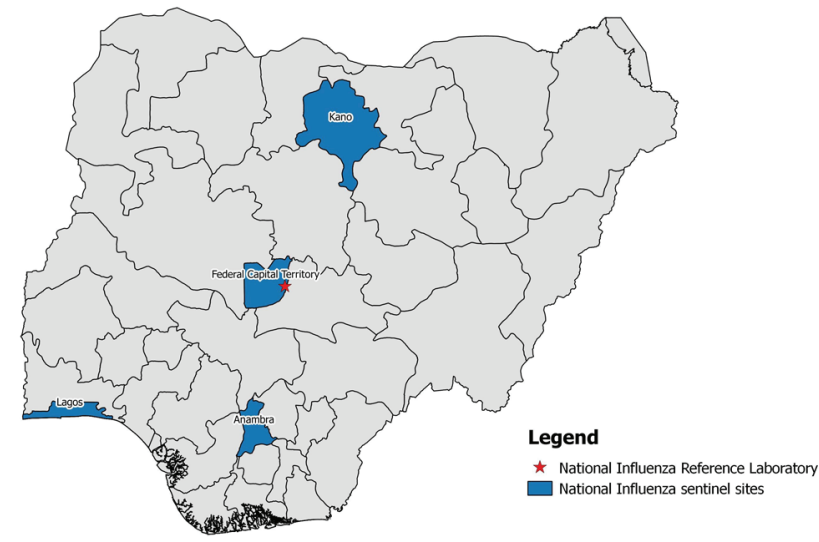

Map of Nigeria showing National influenza sentinel sites and reference laboratory

\section{Keywords}

sentinel surveillance; influenza; Nigeria

\section{Acknowledgments}

We wish to express our gratitute to the National Influenza Sentinel Surveillance team and other stakeholders who made this evaluation possible

\section{References}

1. Federal Ministry of Health (NG).Protocol for National Influenza Sentinel Surveillance. Rev. ed. 2011

2. Centers for Disease Control and Prevention (US). Updated guidelines for evaluating public health surveillance systems: recommendations from the guidelines working group. MMWR 2001;50(No. RR-13)

\section{*Amaka P. Onyiah}

E-mail: pamelaonyiah@yahoo.co.uk 